\title{
ON SPINES OF 3-MANIFOLDS WITH BOUNDARY
}

\author{
C. MCA. GORDON
}

(Received 5 August 1992)

Communicated by J. H. Rubinstein

\begin{abstract}
We give a simple necessary and sufficient condition for the inclusion map of a subpolyhedron into a compact 3-manifold with non-empty boundary to be a homotopy equivalence.

1991 Mathematics subject classification (Amer. Math. Soc.): 57 N 10.
\end{abstract}

\section{Introduction}

In this note we prove the following theorem.

THEOREM 1. Let $Y$ be a compact, connected (triangulated) 3-manifold with $\partial Y \neq \emptyset$, and let $X$ be a connected subpolyhedron of $Y$ such that the maps $\pi_{1}(X) \rightarrow \pi_{1}(Y)$ and $H_{2}(X) \rightarrow H_{2}(Y)$, induced by inclusion, are isomorphisms. Then the inclusion map $X \subset Y$ is a homotopy equivalence.

This has the following corollary.

COROLLARY 2. Let $M$ be a rational homology 3-sphere, and let $Q \subset P \subset M$ be polyhedra such that

(1) each component of $P$ contains exactly one component of $Q$;

(2) each component of $M-Q$ contains exactly one component of $M-P$;

(3) for each $q \in Q$, inclusion induces an isomorphism $\pi_{1}(Q, q) \rightarrow \pi_{1}(P, q)$. Then $Q$ is a deformation retract of $P$.

(C) 1993 Australian Mathematical Society 0263-6115/93\$A2.00+0.00 
Taking $M=S^{3}$ in Corollary 2 gives the result that was announced as Proposition 3.4 in [3].

Theorem 1 is a straightforward consequence of the following result, which is implicit in [1].

THEOREM 3. Let $(K, L)$ be a pair of connected $\mathrm{CW}$ complexes such that $K-L$ has finitely many cells, each of dimension $\leq 2$. Suppose that the maps $\pi_{1}(L) \rightarrow \pi_{1}(K)$ and $H_{2}(L) \rightarrow H_{2}(K)$, induced by inclusion, are isomorphisms. Then the inclusion map $L \subset K$ is a homotopy equivalence.

We learned about the question answered by Corollary 2 in 1988, from $\mathrm{T}$. Y. Kong, who was interested in it in the context of image thinning algorithms for 3-dimensional binary digital images in computer graphics (see [3]). Our original proof of Theorem 1, obtained in 1989, used 3-manifold topology, for example the sphere theorem. Later, on wondering whether the corresponding statement was true in the category of 2-complexes, we were led to Cohen's paper [1] and the realization that it essentially contained a proof of Theorem 3.

I would like to thank Dr. Kong for bringing the question mentioned above to my attention.

\section{Proofs}

PROOF OF THEOREM 3. Since this is not stated explicitly in [1], we describe the relevant parts of that paper and how they imply the theorem. We follow closely the notation of [1].

We may assume that $L$ has a single 0 -cell, $e^{0}$, and that $K-L$ consists of 1-cells and 2-cells. The homology exact sequence of the pair $(K, L)$ shows that $H_{1}(K, L)=H_{2}(K, L)=0$. It follows that the boundary homomorphism $\partial: C_{2}(K, L) \rightarrow C_{1}(K, L)$ is an isomorphism, and hence that $K-L$ has the same number of 1-cells as 2-cells. So $K=L \cup \bigcup_{j=1}^{n} e_{j}^{1} \cup \bigcup_{i=1}^{n} e_{i}^{2}$, say.

Let $L^{*}=L \cup \bigcup_{j=1}^{n} e_{j}^{1}$. Taking $e^{0}$ as base point for $\pi_{1}$ throughout, let $x_{j}$ be the element of $\pi_{1}\left(L^{*}\right)$ represented by $e_{j}^{1}, 1 \leq j \leq n$, and let $F$ be the free group on $\left\{x_{1}, \ldots, x_{n}\right\}$. Then $\pi_{1}\left(L^{*}\right)=\pi_{1}(L) * F$.

Write $G=\pi_{1}(L)$. Let $r_{i} \in G * F$ be the element represented by the attaching map of $e_{i}^{2}, \quad(1 \leq i \leq n)$, and let $R \subset G * F$ be the normal closure of $\left\{r_{1}, \ldots, r_{n}\right\}$. Then $\pi_{1}(K) \cong(G * F) / R=H$, say, where the map $\pi_{1}(L) \rightarrow$ $\pi_{1}(K)$ corresponds to the composition $\varphi: G \subset G * F \rightarrow H$. 
By hypothesis, $\varphi$ is an isomorphism. In particular, since $\varphi$ is onto, there exists $w_{j} \in G$ such that $x_{j} w_{j}^{-1} \in R, \quad(1 \leq j \leq n)$. Let $R_{0} \subset G * F$ be the normal closure of $\left\{x_{1} w_{1}^{-1}, \ldots, x_{n} w_{n}^{-1}\right\}$. Thus $R_{0} \subset R$.

Clearly the composition $\varphi_{0}: G \subset G * F \rightarrow(G * F) / R_{0}=H_{0}$ is an isomorphism. But if $\pi: H_{0} \rightarrow H$ is the quotient map, then $\varphi=\pi \varphi_{0}$. Hence $\pi$ is an isomorphism, giving $R_{0}=R$. Therefore $r_{i} \in R_{0}$, so we may write

$$
r_{i}=\prod_{k=1}^{q_{i}} g_{i k}\left(x_{i k} w_{i k}^{-1}\right)^{n_{i k}} g_{i k}^{-1}, \quad 1 \leq i \leq n,
$$

as in the hypothesis of Lemma 2.3 of [1]. (Here $g_{i k} \in G, n_{i k} \in \mathbb{Z}, x_{i k}=x_{j}$ for some $j$, and $w_{i k}=w_{j}$ for the same $j$.)

Let $p: \tilde{K} \rightarrow K$ be the universal cover. Since $\pi_{1}(L) \rightarrow \pi_{1}(K)$ is an isomorphism, $p^{-1}(L)=\tilde{L}$ is the universal cover of $L$. Note that $C_{q}(\tilde{K}, \tilde{L})=0$ for $q \neq 1,2$, while $C_{1}(\tilde{K}, \tilde{L})$ and $C_{2}(\tilde{K}, \tilde{L})$ are free $\mathbb{Z} G$-modules of rank $n$, with bases corresponding to the 1-cells and 2-cells of $K-L$ respectively. Note also that under the maps $\pi_{1}\left(L^{*}\right) \rightarrow \pi_{1}(K)$ and $\pi_{1}(L) \rightarrow \pi_{1}(K)$ induced by inclusion, $x_{j}$ and $w_{j}(1 \leq j \leq n)$ have the same image. Hence Lemma 2.3 of [1] applies to show that the boundary homomorphism $\partial: C_{2}(\tilde{K}, \tilde{L}) \rightarrow C_{1}(\tilde{K}, \tilde{L})$ is represented, with respect to the bases mentioned above, by the $n \times n$ matrix $A=\left(a_{i j}\right)$ over $\mathbb{Z} G$ defined by

$$
a_{i j}=\sum n_{i k} g_{i k},
$$

where the sum is taken over those $k$ for which $x_{i k}=x_{j}$.

Next, recall the expression for $r_{i}$ given above and define $r_{i}^{\prime} \in G * F$ by the corresponding expression

$$
r_{i}^{\prime}=\prod_{k=1}^{q_{i}} g_{i k} x_{i k}^{n_{i k}} g_{i k}^{-1}, \quad 1 \leq i \leq n,
$$

as in $[1, \S 1]$. Let $R^{\prime} \subset G * F$ be the normal closure of $\left\{r_{1}^{\prime}, \ldots, r_{n}^{\prime}\right\}$.

Let $\alpha: G * F \rightarrow G * F$ be the isomorphism defined by $\alpha \mid G=$ identity and $\alpha\left(x_{i}\right)=x_{i} w_{i}^{-1}, \quad(1 \leq i \leq n)$. Then $\alpha\left(r_{i}^{\prime}\right)=r_{i}, \quad(1 \leq i \leq n)$, so $\alpha\left(R^{\prime}\right)=R$ and $\alpha$ induces an isomorphism $\bar{\alpha}: H^{\prime}=(G * F) / R^{\prime} \rightarrow(G * F) / R=H$. Let $\varphi^{\prime}$ be the composition $G \subset G * F \rightarrow H^{\prime}$. Then $\varphi=\bar{\alpha} \varphi^{\prime}$. Since $\varphi$ is an isomorphism, $\varphi^{\prime}$ is also. Hence, by Proposition 4.1 of [1], the matrix $A$ is invertible.

Thus $\partial: C_{2}(\tilde{K}, \tilde{L}) \rightarrow C_{1}(\tilde{K}, \tilde{L})$ is an isomorphism, and we have $H_{*}(\tilde{K}, \tilde{L})=$ 0 , hence $\pi_{*}(\tilde{K}, \tilde{L})=0$, and hence $\pi_{*}(K, L)=0$, as in [1, Lemma 2.2]. The result follows. 
PROOF OF THEOREM 1. Adding a collar to $\partial Y$ and replacing $X$ by a regular neighborhood, we may assume that $X$ is a compact 3-manifold in the interior of $Y$.

We claim that each component of $\overline{Y-X}$ meets $\partial Y$. For if $Z$ is a component that does not, then $[\partial Z]=0$ in $H_{2}\left(Y ; \mathbb{Z}_{2}\right)$. But $\partial Z$ must consist of a proper subset of the components of $\partial X$, otherwise $Y=X \cup_{\partial} Z$ and hence $\partial Y=\emptyset$, contrary to hypothesis. Therefore $[\partial Z] \neq 0$ in $H_{2}\left(X ; \mathbb{Z}_{2}\right)$. But the universal coefficient theorem shows that the map $H_{2}\left(X ; \mathbb{Z}_{2}\right) \rightarrow H_{2}\left(Y ; \mathbb{Z}_{2}\right)$ is an isomorphism.

Hence, starting at $\partial Y$, we may collapse away all the 3-simplexes of $\overline{Y-X}$, thereby collapsing $Y$ onto $X \cup K$ where $K$ is a finite 2-complex. The result now follows from Theorem 3 .

PROOF OF COROLLARY 2. Since $M$ is a rational homology sphere, $H^{1}(M)=$ 0 , and the cohomology exact sequence of the pair $(M, M-P)$ gives an exact sequence

$$
H^{0}(M) \rightarrow H^{0}(M-P) \rightarrow H^{1}(M, M-P) \rightarrow 0,
$$

and similarly for $Q$.

Condition (2) implies that the map $H^{0}(M-Q) \rightarrow H^{0}(M-P)$ induced by inclusion is an isomorphism. Hence so is the map $H^{1}(M, M-Q) \rightarrow$ $H^{1}(M, M-P)$. It follows, by Alexander Duality, that $H_{2}(Q) \rightarrow H_{2}(P)$ is an isomorphism.

Now replace $P$ by a regular neighborhood $Y$ in $M$, and apply Theorem 1 to each component of $Y$ (with $X$ the corresponding component of $Q$ ).

\section{Concluding Remarks}

Here are two questions related to the above discussion. Let $X$ and $Y$ be either finite connected 2-complexes or compact connected 3-manifolds with non-empty boundary.

(1) If $f: X \rightarrow Y$ is a map inducing isomorphisms on $\pi_{1}$ and $H_{2}$, is $f$ a homotopy equivalence?

(2) If $\pi_{1}(X) \cong \pi_{1}(Y)$ and $H_{2}(X) \cong H_{2}(Y)$, are $X$ and $Y$ homotopy equivalent?

Theorems 3 and 1 show that the answer to (1) is 'yes' in both cases if $f$ is an inclusion map. On the other hand, it is easy to construct counterexamples in general. (For example, take $X=Y=S^{1} \times S^{2}$-open 3-cell $\simeq S^{1} \vee S^{2}$. Then 
$\pi_{1}(X) \cong \mathbb{Z}$, generated by $z$, say, and $\pi_{2}(X) \cong \mathbb{Z} \pi_{1}(X)$, generated by $x$, say. Define $f: X \rightarrow X$ so that $f_{*}(z)=z$ and $f_{*}(x)=\left(1-z+z^{2}\right) x$.)

Question (2) for finite 2-complexes has been extensively investigated. The answer is 'no' in general; counterexamples were first given by Dunwoody [2] and Metzler [4]. In fact, in the example given in [2], $X$ is homotopy equivalent to the exterior of the trefoil knot minus an open 3-cell. One can show, however, that the answer to (2) is 'yes' in the case of 3-manifolds with boundary.

\section{References}

[1] M. M. Cohen, 'Whitehead torsion, group extensions, and Zeeman's conjecture in high dimensions', Topology 16 (1977), 79-88.

[2] M. J. Dunwoody, 'The homotopy type of a two-dimensional complex', Bull. London Math. Soc. 8 (1976), 282-285.

[3] T. Y. Kong, R. Litherland and A. Rosenfeld, 'Problems in the topology of binary images', in: Open Problems in Topology (eds. J. van Mill and G. M. Reed) (North Holland, 1990).

[4] W. Metzler, 'Über den Homotopietyp zweidimensionaler $C W$-Komplexe und Elementartransformationen bei Darstellungen von Gruppen durch Erzeugende und definierende Relationen', J. reine angew. Math. 285 (1976), 7-23.

Department of Mathematics

The University of Texas at Austin

Austin, TX 78712-1082

U.S.A. 